\title{
Efficient lattice constants and energy band gaps for condensed systems from a meta-GGA level screened range separated hybrid functional
}

\author{
Subrata Jana, ${ }^{1, *}$ Abhilash Patra, ${ }^{1}$ and Prasanjit Samal ${ }^{1}$ \\ ${ }^{1}$ School of Physical Sciences, National Institute of Science Education and Research, HBNI, Bhubaneswar 752050, India
}

(Dated: April 5, 2022)

\begin{abstract}
A meta generalized gradient level screened range-separated hybrid functional is developed for solid-state electronic structure theory. Assessment of the present range-separated hybrid functional for solid-state lattice constants and band gaps indicate that the present functional can be used for describing those properties efficiently in meta-GGA level. Specifically, the performance of the present functional for band gap of solids indicates that the present meta-GGA level screened hybrids functional is quite productive beyond the GGA level. The most appealing feature of the present formalism is that a method has been suggested which is based upon an accurate semilocal functional.
\end{abstract}

\section{INTRODUCTION}

The Kohn-Sham (KS) formalism of density functional theory (DFT) $)^{1} \underline{2}$ is one of the most widely used and accurate theoretical framework for electronic structure calculations of condensed systems. In the $\mathrm{KS}$ formalism the system is effectively one electron like where all the many electron interactions are taken care by exchangecorrelation (XC) energy or potential. The effective one electron potential in DFT, also best known as KS potential is sum of the classical coulomb or Hartree potential $\left(v_{H}\right)$, the exchange-correlation potential $\left(v_{x c}\right)$, and the external potential generated by the nuclei (or ion) $\left(v_{\text {ext }}\right)$ :

$$
v_{\mathrm{KS}}(\mathbf{r})=v_{H}(\mathbf{r})+v_{x c}(\mathbf{r})+v_{e x t}(\mathbf{r}) .
$$

In Eq.(1) the only unknown quantity is the $v_{x c}$, which need to be treated approximately in DFT. Various approximations $s^{3-26,39}$ are proposed for last couple of decades to treat accurately the $v_{x c}$. All these approximations are recognized through the Jacob's ladder ${ }^{3}$, where each rung of the ladder add an extra ingredients starting from local density approximations ${ }^{4}$. The generalized gradient approximations (GGA) $)^{\underline{-16}}$ and meta generalized gradient approximations (meta-GGA) ${ }^{17-26}$ are next two higher rung after LDA. The LDA, GGA and metaGGA are widely used for performing electronic structure calculations $^{27}-37$ in DFT community for their semilocal nature. though the semilocal approximations enjoy early success but due to lack of "many electron self interaction (MESI)" and "non-locality" 38 there are cases in which the performance of semilocal approximations is not satisfactory. The non-locality within the density functionals approximations (DFAs) are introduced through the mixing of Hartree-Fock (HF) exact-exchange either globally (global hybrids) ${ }^{39-41}$ or in range separated scheme (range separated hybrids $)^{42-50}$. Though the global hybrid functionals are very popular in quantum chemistry ${ }^{39-41}$ but they are not so popular for condensed matter electronic structure theory ${ }^{51}$. Beyond the global and long range corrected hybrid functionals, the range separated hybrids proposed using short range HF with DFAs are very popular due to their very improved performance for the solid state systems, especially in band gaps $42,43,52-77$.
Designing a range separated hybrid functionals requires the exchange hole. The exchange hole is proposed using Taylor series approximations ${ }^{17}$ or density matrix expansion 26 or reversed engineered technique $46,78-80$. The popular Heyd-Scuseria-Ernzerhof (HSE) ${ }^{42,43}$ functional is designed using the reversed engineered exchange hole of PBE functional. Beyond the GGA level screened range separated hybrid functionals, the meta-GGA level screened range separated hybrid functionals is also proposed recently by Tao et.al $\stackrel{46}{*}$ using by utilizing the TPSS exchange energy functionals. Beyond the TPSS exchange energy funcional, very recently, Tao-Mo proposed an accurate semilocal functional 26 for quantum chemistry and solid state system using density matrix expansion based semilocal exchange hole with the slowly varying fourth order gradient approximation. Motivated by the TM functional and its underlying construction we propose an screened range separated hybrid functional to be used for condensed matter systems. In designing the present screened range separated hybrid functional we utilize the local density approximation based exchange hole for the short range semilocal part with the short range HF. This is the possible conventional way to utilize the TM functional in screened range separated hybrid functional scheme for solid state systems bypassing its reversed engineered exchange hole. Though, very recently, another way of inclusion of exchange hole in short range semilocal functional is proposed ${ }^{50}$, but, that scheme not perform satisfactory way as it is found in the present work. Surprisingly, the only LDA exchange hole on the top of the TM exchange-correlation functional performs efficiently in describing both the lattice constants and band gaps of solids. In this paper we design a screened range separated hybrid functional in meta-GGA level and the performance of the present functional is carried out for solid state lattice constants and band gaps using the projectoraugmented-wave method ${ }^{81-86}$ with the plane wave basis set.

The present paper is organized as follows: In the following we will discuss about the generalized KS potential to be used in the hybrid functional calculations. Following this we will give the formulation of the present range separated hybrid functional using semilocal ex- 
change functional and short range HF. Next we will briefly discuss the implementation of the developed range separated functional and its performance for solid state lattice constants and band gaps.

\section{METHODOLOGY}

The general scheme of inclusion of non-local XC potential within the KS formalism is known as generalized Kohn-Sham formalism (gKS). The gKS potential is written as,

$$
\begin{aligned}
v_{x c}\left(\mathbf{r}, \mathbf{r}^{\prime}\right)=\alpha v_{x}^{H F-s r}\left(\mathbf{r}, \mathbf{r}^{\prime} ; \mu\right)+ & (1-\alpha) v_{x}^{s l-s r, \mu} \\
& +v_{x}^{s l-l r, \mu}+v_{c}^{s l} .
\end{aligned}
$$

Alternatively, this can be written as,

$$
v_{x c}\left(\mathbf{r}, \mathbf{r}^{\prime}\right)=\alpha v_{x}^{H F-s r}\left(\mathbf{r}, \mathbf{r}^{\prime} ; \mu\right)-\alpha v_{x}^{s l-s r, \mu}+v_{x}^{s l}+v_{c}^{s l},
$$

where the semilocal short range (sl-sr) and semilocal long range (sl-lr) part added into the semilocal exchangecorrelation functional which in our present case is the TM functional. Here, the parameter $\alpha$ controls the amount of HF mixs with the semilocal functional and $\mu$ is the range separated parameter. The $\alpha=0$ value corresponds to pure semilocal formalism.

The range-separated density functional theory is actually developed by separating the $\frac{1}{\left|\mathbf{r}-\mathbf{r}^{\prime}\right|}$ operator into short and long-range part as,

$$
\frac{1}{\left|\mathbf{r}-\mathbf{r}^{\prime}\right|}=\underbrace{\frac{\operatorname{Erf}\left(\mu\left|\mathbf{r}-\mathbf{r}^{\prime}\right|\right)}{\left|\mathbf{r}-\mathbf{r}^{\prime}\right|}}_{l r}+\underbrace{\frac{\operatorname{Erf}\left(\mu\left|\mathbf{r}-\mathbf{r}^{\prime}\right|\right)}{\left|\mathbf{r}-\mathbf{r}^{\prime}\right|}}_{s r} .
$$

Using the above seperation scheme the range separated parameter $\mu$ of Eq.(3) is included into the exact HF exchange through the following equation,

$$
v_{x}^{H F-s r}\left(\mathbf{r}, \mathbf{r}^{\prime} ; \mu\right)=-\sum_{i=1}^{\mathrm{occ}} \phi_{i}(\mathbf{r}) \frac{\operatorname{Erfc}\left(\mu\left|\mathbf{r}-\mathbf{r}^{\prime}\right|\right)}{\left|\mathbf{r}-\mathbf{r}^{\prime}\right|} \phi_{i}^{*}\left(\mathbf{r}^{\prime}\right),
$$

where $\phi_{i}$ s are single particle electronic orbitals. Except screened HF exchange, other unknown potentials of Eq.(3) are the screened potential $\left(v_{x}^{s l-s r, \mu}\right)$ and the semilocal potential $\left(v_{x}^{s l}\right)$. In meta-GGA level theory the exchange potential is obtained not only by taking the derivative with respect of density and gradient of density of the exchange energy functional but also the partial derivative of KS kinetic energy density is also required. In gKS formalism the semilocal exchange potential is expressed as

$$
\begin{aligned}
v_{x}^{s l} \Psi_{i} & =\left[\frac{\partial\left(\rho \epsilon_{x}^{s l}\right)}{\partial \rho}-\vec{\nabla} \frac{\partial\left(\rho \epsilon_{x}^{s l}\right)}{\partial \vec{\nabla} \rho}\right] \Psi_{i}-\frac{1}{2} \vec{\nabla}\left(\frac{\partial\left(\rho \epsilon_{x}^{s l}\right)}{\partial \tau}\right) \vec{\nabla} \Psi_{i} \\
& -\frac{1}{2} \frac{\partial\left(\rho \epsilon_{x}^{s l}\right)}{\partial \tau} \vec{\nabla}^{2} \Psi_{i}
\end{aligned}
$$

where $\epsilon_{x}^{s l}$ is the exchange energy density. In our present study $\epsilon_{x}^{s l}$ is the TM semilocal exchange energy density. The TM exchange energy functional can be expressed as,

$$
\begin{aligned}
E_{x}^{T M} & =-\int d \mathbf{r} \rho(\mathbf{r}) \epsilon_{x}^{u n i f} F_{x}^{T M}, \\
& =-\int d \mathbf{r} \rho(\mathbf{r}) \epsilon_{x}^{s l} .
\end{aligned}
$$

The TM enhancement factor is given by,

$$
F_{x}^{T M}=w F_{x}^{D M E}+(1-w) F_{x}^{s c},
$$

where, $F_{x}^{D M E}=1 / f^{2}+7 R /\left(9 f^{4}\right)$ is the enhancement factor derived from density matrix expansion. Here, $R=1+595(2 \lambda-1)^{2} p / 54-\left[\tau-\left(3 \lambda^{2}-\lambda+1 / 2\right)\left(\tau-\tau^{\text {unif }}-\right.\right.$ $\left.\left.\left.|\nabla \rho|^{2} /(72 \rho)\right)\right] / \tau^{\text {unif }}\right), f=\left[1+10(70 y / 27)+\beta y^{2}\right]^{1 / 10}$ (with $y=(2 \lambda-1)^{2} p$ ) and the slowly varying fourth order gradient expansion is given by $F_{x}^{s c}=\left[1+10\left\{\left(\frac{10}{81}+\right.\right.\right.$ $\left.\left.\left.\frac{50 p}{729}\right) p+\frac{146}{2025} \tilde{q}^{2}-\left(\frac{73 \tilde{q}}{405}\right)\left[\frac{3 \tau^{w}}{5 \tau}\right]\left(1-\frac{\tau^{w}}{\tau}\right)\right\}\right]^{\frac{1}{10}}$. The TM functional used $w$ as the weight factor between DME based functional form and slowly varying fourth order gradient expansion. The readers are suggested to go through the references $\frac{26,32}{}$ for the details of the derivation of the functional form and the terms associated with the TM functional. The interpolation factor $w$ is the function of meta-GGA ingredient $z=\tau_{W} / \tau$, where $\tau_{W}$ is the von Weizsäcker kinetic energy density. In the slowly varying density limit $w$ is small therefore the fourth order density gradient approximation dominates. Not only that the interpolation factor $w$ have different unique features which makes the TM functionals works equally well both for molecular and solid state systems. The semilocal potential of the TM functional can be derived from Eq. (6). Now, only the remaining part of the potential is the semilocal short-range part. In the present case we have constructed the semilocal short-range from the LDA exchange hole. Using the LDA exchange hole the semilocal short range part of the exchange energy functional becomes,

$$
\begin{aligned}
E_{x}^{s l-s r, \mu}=-\int & d \mathbf{r} \rho(\mathbf{r}) \epsilon_{x}^{u n i f}\left\{1-\frac{8}{3} \mathcal{A}\left(\sqrt{\pi} \operatorname{erf}\left(\frac{1}{2 \mathcal{A}}\right)\right.\right. \\
& \left.\left.+\left(2 \mathcal{A}-4 \mathcal{A}^{3}\right) e^{-\frac{1}{4 \mathcal{A}^{2}}}-3 \mathcal{A}+4 \mathcal{A}^{3}\right)\right\},
\end{aligned}
$$

where $\epsilon_{x}^{u n i f}=\frac{3 k_{f}}{4 \pi}$ is the exchange energy per electron of the homogeneous electron gas and $\mathcal{A}=\frac{\mu}{2 k_{f}}$. Through $\mathcal{A}$ the screening parameter includes into the semilocal short range part. The reason we use only LDA exchange hole in our semilocal short range part because, using it we obtain satisfactory results for both the lattice constant and band gaps. Other way the inclusion of exchange hole is given in reference ${ }^{50}$. But the inclusion of full scheme (given in reference ${ }^{50}$ ) into this present functional form worsen its performance for lattice constants. Therefore, we stick with the LDA exchange hole only. The 
TABLE I. Equilibrium lattice constant $a_{0}$ (in $\AA$ ) of different solid structures using HSE06, SRSH-TM-TPSSc and SRSH-TM. All the experimental reference values are collected from ref $.32,55$. The structures we consider here are A1 = face-centered cubic, $\mathrm{A} 2=$ diamond, A3 = body-centered cubic, B3 = zinc blende, and B1 = rock salt. The relative deviation (in percentage) of the individual species using each functional is also given.

\begin{tabular}{|c|c|c|c|c|c|c|c|}
\hline$\overline{\text { Solids }}$ & $\overline{\mathrm{HSE}} 66$ & $\overline{\%}$ & $\overline{\text { SRSH-TM-TPSSc }}$ & $\%$ & $\overline{\overline{\text { SRSH-TM }}}$ & $\%$ & Expt. \\
\hline $\mathrm{C}(\mathrm{A} 2)$ & 3.548 & -0.53 & 3.550 & -0.48 & 3.545 & -0.62 & 3.567 \\
\hline Si (A2) & 5.432 & 0.04 & 5.420 & -0.18 & 5.408 & -0.40 & 5.430 \\
\hline $\mathrm{Ge}(\mathrm{A} 2)$ & 5.676 & 0.42 & 5.653 & 0.02 & 5.636 & -0.28 & 5.652 \\
\hline $\mathrm{SiC}(\mathrm{B} 3)$ & 4.346 & -0.27 & 4.338 & -0.46 & 4.332 & -0.59 & 4.358 \\
\hline $\mathrm{BN}(\mathrm{B} 3)$ & 3.597 & -0.28 & 3.603 & -0.11 & 3.597 & -0.28 & 3.607 \\
\hline $\mathrm{BP}(\mathrm{B} 3)$ & 4.519 & -0.42 & 4.52 & -0.40 & 4.509 & -0.64 & 4.538 \\
\hline BAs (B3) & 4.770 & -0.15 & 4.766 & -0.23 & 4.754 & -0.48 & 4.777 \\
\hline $\mathrm{BSb}$ (B3) & 5.216 & $\mathrm{n} / \mathrm{a}$ & 5.202 & $\mathrm{n} / \mathrm{a}$ & 5.188 & $\mathrm{n} / \mathrm{a}$ & $\mathrm{n} / \mathrm{a}$ \\
\hline AlP (B3) & 5.470 & 0.18 & 5.461 & $0 . .02$ & 5.448 & -0.22 & 5.460 \\
\hline AlAs (B3) & 5.676 & 0.32 & 5.659 & 0.02 & 5.646 & -0.21 & 5.658 \\
\hline AlSb (B3) & 6.151 & 0.24 & 6.130 & -0.10 & 6.114 & -0.36 & 6.136 \\
\hline$\beta-\mathrm{GaN}(\mathrm{B} 3)$ & 4.521 & -0.22 & 4.526 & -0.11 & 4.516 & -0.33 & 4.531 \\
\hline $\mathrm{GaP}(\mathrm{B} 3)$ & 5.464 & 0.29 & 5.463 & 0.27 & 5.446 & -0.03 & 5.448 \\
\hline GaAs (B3) & 5.667 & 0.34 & 5.653 & 0.09 & 5.635 & -0.23 & 5.648 \\
\hline GaSb (B3) & 6.099 & 0.05 & 6.075 & -0.34 & 6.055 & -0.67 & 6.096 \\
\hline $\mathrm{InP}(\mathrm{B} 3)$ & 5.921 & 0.94 & 5.923 & 0.68 & 5.903 & 0.63 & 5.866 \\
\hline InAs (B3) & 6.108 & 0.89 & 6.095 & 0.68 & 6.075 & 0.34 & 6.054 \\
\hline InSb (B3) & 6.516 & 0.57 & 6.496 & 0.26 & 6.473 & -0.09 & 6.479 \\
\hline $\mathrm{ZnS}$ (B3) & 5.419 & 0.18 & 5.436 & 0.50 & 5.412 & 0.05 & 5.409 \\
\hline ZnSe (B3) & 5.693 & 0.44 & 5.699 & 0.55 & 5.676 & 0.14 & 5.668 \\
\hline ZnTe (B3) & 6.135 & 0.75 & 6.129 & 0.65 & 6.099 & 0.16 & 6.089 \\
\hline CdS (B3) & 5.880 & 1.06 & 5.924 & 1.82 & 5.893 & 1.29 & 5.818 \\
\hline CdSe (B3) & 6.133 & 1.34 & 6.164 & 1.85 & 6.133 & 1.34 & 6.052 \\
\hline CdTe (B3) & 6.543 & 0.97 & 6.568 & 1.36 & 6.533 & 0.81 & 6.480 \\
\hline $\mathrm{MgO}(\mathrm{B} 1)$ & 4.197 & -0.24 & 4.195 & -0.28 & 4.187 & -0.47 & 4.207 \\
\hline MgS (B3) & 5.652 & 8.65 & 5.647 & 8.55 & 5.632 & 8.27 & 5.202 \\
\hline MgSe (B1) & 5.454 & 1.00 & 5.462 & 1.15 & 5.411 & 0.76 & 5.400 \\
\hline MgTe (B3) & 6.452 & 0.50 & 6.446 & 0.40 & 6.424 & 0.06 & 6.420 \\
\hline $\mathrm{CaS}(\mathrm{B} 1)$ & 5.698 & 0.16 & 5.722 & 0.58 & 5.699 & 0.17 & 5.689 \\
\hline CaSe (B1) & 5.938 & 0.37 & 5.966 & 0.84 & 5.939 & 0.38 & 5.916 \\
\hline CaTe (B1) & 6.369 & 0.33 & 6.404 & 0.88 & 6.369 & 0.33 & 6.348 \\
\hline $\mathrm{SrS}(\mathrm{B} 1)$ & 6.034 & 0.73 & 6.071 & 1.35 & 6.046 & 0.93 & 5.990 \\
\hline SrSe (B1) & 6.268 & 0.54 & 6.302 & 1.09 & 6.275 & 0.66 & 6.234 \\
\hline SrTe (B1) & 6.684 & 0.66 & 6.721 & 1.22 & 6.688 & 0.72 & 6.640 \\
\hline $\mathrm{BaS}(\mathrm{B} 1)$ & 6.432 & 0.67 & 6.487 & 1.53 & 6.454 & 1.02 & 6.389 \\
\hline BaSe (B1) & 6.656 & 0.92 & 6.707 & 1.70 & 6.673 & 1.18 & 6.595 \\
\hline $\mathrm{BaTe}(\mathrm{B} 1)$ & 7.057 & 0.71 & 7.115 & 1.54 & 7.075 & 0.97 & 7.007 \\
\hline $\mathrm{Ag}(\mathrm{A} 1)$ & 4.146 & 1.89 & 4.151 & 2.02 & 4.135 & 1.62 & 4.069 \\
\hline $\mathrm{Al}(\mathrm{A} 1)$ & 4.020 & -0.30 & 3.980 & -1.29 & 3.979 & -1.31 & 4.032 \\
\hline $\mathrm{Cu}(\mathrm{A} 1)$ & 3.637 & 0.94 & 3.573 & -0.83 & 3.573 & -0.83 & 3.603 \\
\hline $\mathrm{Pd}(\mathrm{A} 1)$ & 3.904 & 0.59 & 3.923 & 1.08 & 3.909 & 0.72 & 3.881 \\
\hline $\mathrm{K}(\mathrm{A} 3)$ & 5.32 & 1.82 & 5.297 & 1.38 & 5.273 & 0.92 & 5.225 \\
\hline Li (A3) & 3.466 & -0.32 & 3.439 & -1.09 & 3.440 & -1.06 & 3.477 \\
\hline $\mathrm{LiCl}$ (B1) & 5.116 & 0.19 & 5.100 & -0.12 & 5.076 & -0.59 & 5.106 \\
\hline $\mathrm{LiF}(\mathrm{B} 1)$ & 4.015 & 0.12 & 3.973 & -0.92 & 3.968 & -1.05 & 4.010 \\
\hline $\mathrm{NaCl}(\mathrm{B} 1)$ & 5.613 & 0.32 & 5.556 & -0.70 & 5.540 & -0.98 & 5.595 \\
\hline $\mathrm{NaF}(\mathrm{B} 1)$ & 4.576 & -0.71 & 4.513 & -2.08 & 4.507 & -2.21 & 4.609 \\
\hline
\end{tabular}

semilocal short range potential of the present functional form can also be obtained from Eq.(6). This completes the semilocal exchange potential of the present range separated functional. For the correlation we have used one electron self-interaction free Tao-Perdew-StaroverovScuseria (TPSS) ${ }^{22}$ and its modified version ${ }^{26}$ for TM exchange in our present study. The TPSS and TM cor- relation gives different results for various properties of solids 32 . Utilizing the present range separated functional coupled with the TPSS and TM correlation therefore give rise two screened range separated functional. We named those as (i) SRSH-TM-TPSS (screened range separated hybrid which uses TM exchange plus TPSS correlation) and (ii) SRSH-TM (screened range separated 


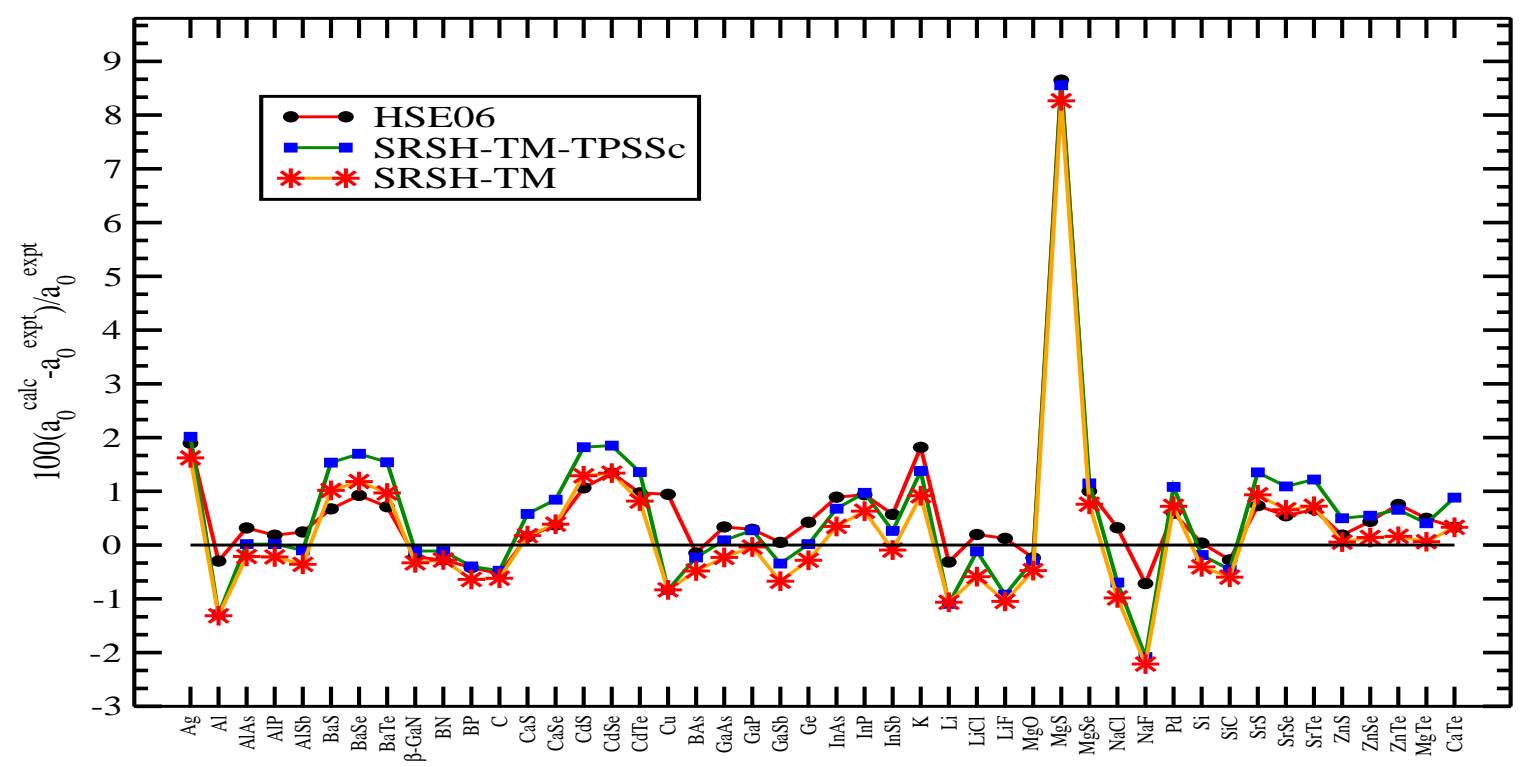

tice constants with respect to the experimental (ZPAE-

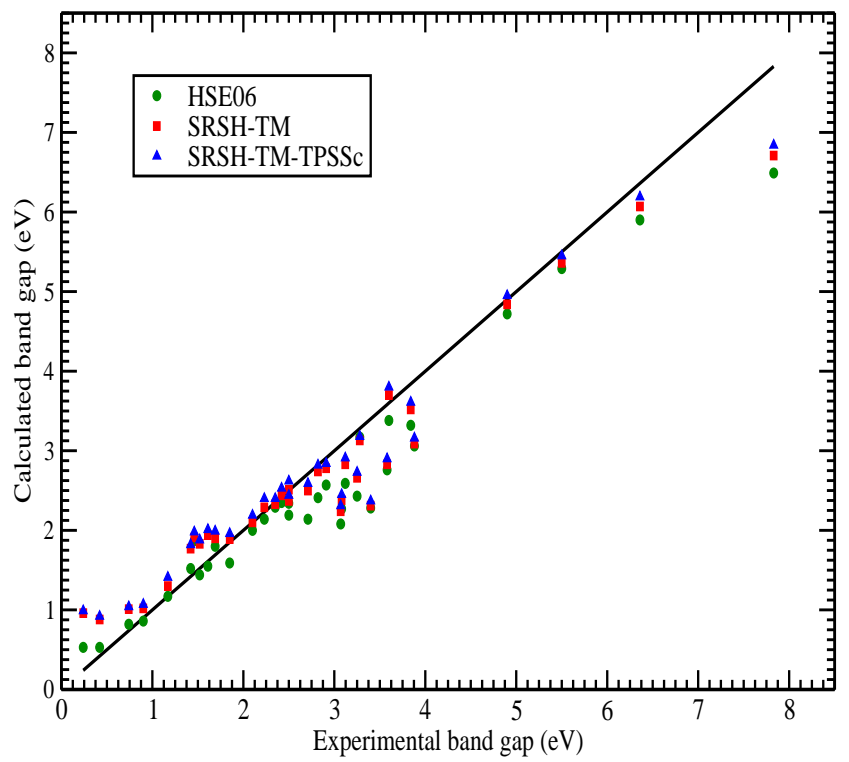

FIG. 2. Calculated versus experimental band gaps for 36 solids presented in Table - II using different functionals.

hybrid which uses TM exchange plus modified TPSS correlation). We assess the performance of both the SRSHTM-TPSS and SRSH-TM for solid state lattice constants and band gaps.

All the self consistence calculations of the present functional is carried in the projector-augmented-wave (PAW) environment with the plane wave basis set in Vienna $\mathrm{Ab}$ initio simulation package (VASP) 1 - 26 . The PAW methods are very accurate in density functional calculations and its performance is same as all electron calculations used in different codes. The TM functional is recently implemented 37 in VASP. Present implementation of range separate hybrid functional is based on this TM implementation.

Now we come to the discussion of the value of $\mu$ and $\alpha$ for our present range separated functional. The value of the parameter $\alpha$ is chosen to be 0.25 . This value is recommended by recently proposed TPSS based meta-GGA hybrid functional and also it has been used in HSE06 functional. Regarding $\mu$ parameter, in the HSE06 functional the $\mu$ parameter is set to $0.11 \mathrm{bohr}^{-1}$, yielding a well balanced description for lattice constants and band gaps. In the present case we also recommended $\mu=0.11$ bohr ${ }^{-1}$ which produce a very balanced treatment of both the lattice constants and band gaps. The performance of the present meta-GGA level range separated functional is compared with widely used HSE06 functional. Unless otherwise stated the default values $\mu$ and $\alpha$ values are used in VASP recommended HSE06 calculations.

Here we have calculated the mean (relative) error (ME/MRE), mean absolute (relative) error (MAE/MARE) and the standard deviation of the (relative) error (STDE/STDRE) to study the accuracy of each functionals. The definition we used here to calcu- 
TABLE II. Band gaps using different functionals calculated at the experimental lattice constants. The structure considered here are $\mathrm{A} 1$ = rock salt, $\mathrm{A} 2=$ diamond, $\mathrm{A} 3=$ zinc blende. Here, the experimental geometries and band gap values are taken from reference ${ }^{73}$.

\begin{tabular}{|c|c|c|c|c|c|c|c|c|c|}
\hline Solids & Space Group & Geometry $(\AA)$ & HSE06 & $\%$ & SRSH-TM-TPSSc & $\%$ & SRSH-TM & $\%$ & Expt. \\
\hline $\mathrm{MgO}(\mathrm{A} 1)$ & $\mathrm{Fm} \overline{3} \mathrm{~m}$ & 4.207 & 6.49 & -17.11 & 6.84 & -12.64 & 6.71 & -14.30 & 7.83 \\
\hline $\mathrm{BaS}(\mathrm{A} 1)$ & $\mathrm{Fm} \overline{3} \mathrm{~m}$ & 6.389 & 3.06 & -21.13 & 3.16 & -18.55 & 3.09 & -20.36 & 3.88 \\
\hline $\mathrm{BaSe}(\mathrm{A} 1)$ & $\mathrm{Fm} \overline{3} \mathrm{~m}$ & 6.595 & 2.76 & -22.90 & 2.91 & -18.99 & 2.83 & -20.94 & 3.58 \\
\hline $\operatorname{BaTe}(\mathrm{A} 1)$ & Fmㅍm & 7.007 & 2.27 & -26.29 & 2.45 & -20.45 & 2.38 & -22.72 & 3.08 \\
\hline $\mathrm{ScN}(\mathrm{A} 1)$ & Fm $\overline{3} \mathrm{~m}$ & 4.500 & 0.86 & -4.44 & 1.07 & 18.88 & 1.02 & 13.33 & 0.9 \\
\hline $\mathrm{AgCl}(\mathrm{A} 1)$ & $\mathrm{Fm} \overline{3} \mathrm{~m}$ & 5.546 & 2.43 & -25.23 & 2.73 & -16.00 & 2.66 & -18.15 & 3.25 \\
\hline $\mathrm{AgBr}(\mathrm{A} 1)$ & $\mathrm{Fm} \overline{3} \mathrm{~m}$ & 5.772 & 2.14 & -21.03 & 2.59 & -4.42 & 2.50 & -7.74 & 2.71 \\
\hline C (A2) & $\mathrm{Fd} \overline{3} \mathrm{~m}$ & 3.567 & 5.29 & -3.81 & 5.45 & -0.90 & 5.36 & -2.54 & 5.5 \\
\hline $\mathrm{Si}(\mathrm{A} 2)$ & $\mathrm{Fd} \overline{3} \mathrm{~m}$ & 5.430 & 1.17 & 0.00 & 1.41 & 20.51 & 1.30 & 11.11 & 1.17 \\
\hline $\mathrm{Ge}(\mathrm{A} 2)$ & $\mathrm{Fd} \overline{3} \mathrm{~m}$ & 5.430 & 0.82 & 10.81 & 1.04 & 40.54 & 1.01 & 36.48 & 0.74 \\
\hline $\mathrm{SiC}$ (A3) & $\mathrm{F} \overline{4} 3 \mathrm{~m}$ & 4.358 & 2.35 & -2.89 & 2.53 & 4.54 & 2.44 & 0.82 & 2.42 \\
\hline $\mathrm{BN}(\mathrm{A} 3)$ & $\mathrm{F} \overline{4} 3 \mathrm{~m}$ & 3.616 & 5.90 & -7.23 & 6.19 & -2.67 & 6.07 & -4.55 & 6.36 \\
\hline $\mathrm{BP}(\mathrm{A} 3)$ & $\mathrm{F} \overline{4} 3 \mathrm{~m}$ & 4.538 & 2.01 & -4.76 & 2.19 & 4.28 & 2.10 & 0.00 & 2.1 \\
\hline BAs (A3) & $\mathrm{F} \overline{4} 3 \mathrm{~m}$ & 4.777 & 1.87 & 28.08 & 1.98 & 35.61 & 1.91 & 30.82 & 1.46 \\
\hline AlN (A3) & $\mathrm{F} \overline{4} 3 \mathrm{~m}$ & 4.342 & 4.72 & -3.67 & 4.95 & 1.02 & 4.84 & -1.22 & 4.9 \\
\hline $\operatorname{AlP}(\mathrm{A} 3)$ & $\mathrm{F} \overline{4} 3 \mathrm{~m}$ & 5.463 & 2.34 & -6.40 & 2.62 & 4.80 & 2.51 & 0.40 & 2.5 \\
\hline AlAs (A3) & $\mathrm{F} \overline{4} 3 \mathrm{~m}$ & 5.661 & 2.15 & -4.03 & 2.40 & 7.62 & 2.29 & 2.69 & 2.23 \\
\hline $\mathrm{AlSb}$ (A3) & $\mathrm{F} \overline{4} 3 \mathrm{~m}$ & 6.136 & 1.81 & 6.50 & 1.99 & 17.75 & 1.90 & 12.42 & 1.69 \\
\hline GaN (A3) & $\mathrm{F} \overline{4} 3 \mathrm{~m}$ & 3.180 & 3.17 & -3.35 & 3.18 & -3.04 & 3.13 & -4.57 & 3.28 \\
\hline $\mathrm{GaP}(\mathrm{A} 3)$ & $\mathrm{F} \overline{4} 3 \mathrm{~m}$ & 5.451 & 2.29 & -2.55 & 2.40 & 2.12 & 2.33 & -0.85 & 2.35 \\
\hline GaAs (A3) & $\mathrm{F} \overline{4} 3 \mathrm{~m}$ & 5.648 & 1.44 & -5.26 & 1.88 & 23.68 & 1.83 & 20.39 & 1.52 \\
\hline $\mathrm{InP}(\mathrm{A} 3)$ & $\mathrm{F} \overline{4} 3 \mathrm{~m}$ & 5.869 & 1.52 & 7.04 & 1.82 & 28.16 & 1.77 & 24.64 & 1.42 \\
\hline InAs (A3) & $\mathrm{F} \overline{4} 3 \mathrm{~m}$ & 6.058 & 0.53 & 26.19 & 0.92 & 119.04 & 0.88 & 109.52 & 0.42 \\
\hline $\mathrm{InSb}(\mathrm{A} 3)$ & $\mathrm{F} \overline{4} 3 \mathrm{~m}$ & 6.479 & 0.53 & 120.83 & 0.99 & 312.50 & 0.96 & 300.00 & 0.24 \\
\hline $\operatorname{MgTe}(\mathrm{A} 3)$ & $\mathrm{F} \overline{4} 3 \mathrm{~m}$ & 6.420 & 3.38 & -6.11 & 3.80 & 5.55 & 3.70 & 2.77 & 3.6 \\
\hline $\mathrm{CuCl}(\mathrm{A} 3)$ & $\mathrm{F} \overline{4} 3 \mathrm{~m}$ & 5.501 & 2.28 & -32.94 & 2.37 & -30.29 & 2.31 & -32.05 & 3.4 \\
\hline $\mathrm{CuBr}$ (A3) & $\mathrm{F} \overline{4} 3 \mathrm{~m}$ & 5.820 & 2.08 & -32.24 & 2.31 & -24.75 & 2.24 & -27.03 & 3.07 \\
\hline $\mathrm{CuI}(\mathrm{A} 3)$ & $\mathrm{F} \overline{4} 3 \mathrm{~m}$ & 6.063 & 2.59 & -16.98 & 2.91 & -6.73 & 2.83 & -9.29 & 3.12 \\
\hline $\mathrm{ZnS}(\mathrm{A} 3)$ & $\mathrm{F} \overline{4} 3 \mathrm{~m}$ & 5.409 & 3.32 & -13.54 & 3.61 & -5.98 & 3.52 & -8.33 & 3.84 \\
\hline ZnSe (A3) & $\mathrm{F} \overline{4} 3 \mathrm{~m}$ & 5.668 & 2.41 & -14.53 & 2.82 & 0.00 & 2.74 & -2.83 & 2.82 \\
\hline AgI (A3) & $\mathrm{F} \overline{4} 3 \mathrm{~m}$ & 6.499 & 2.57 & -11.68 & 2.84 & -2.40 & 2.78 & -4.46 & 2.91 \\
\hline CdS (A3) & $\mathrm{F} \overline{4} 3 \mathrm{~m}$ & 5.818 & 2.19 & -12.40 & 2.45 & -2.40 & 2.37 & -5.20 & 2.5 \\
\hline CdSe (A3) & $\mathrm{F} \overline{4} 3 \mathrm{~m}$ & 6.052 & 1.59 & -14.05 & 1.96 & 5.94 & 1.89 & 2.16 & 1.85 \\
\hline $\mathrm{CdTe}(\mathrm{A} 3)$ & $\mathrm{F} \overline{4} 3 \mathrm{~m}$ & 6.480 & 1.55 & -3.72 & 2.01 & 24.84 & 1.94 & 20.49 & 1.61 \\
\hline
\end{tabular}

TABLE III. Summary statistics for the error in the calculated lattice constants and band gaps for the set of solids presented in Table-I and Table-II.

\begin{tabular}{cccc}
\hline \hline \multicolumn{4}{c}{ HSE06 SRSH-TM-TPSSc SRSH-TM } \\
\hline ME $(\AA)$ & 0.033 & 0.033 & 0.015 \\
MAE $(\AA)$ & 0.039 & 0.052 & 0.042 \\
STDE $(\AA)$ & 0.069 & 0.079 & 0.075 \\
MRE $(\%)$ & 0.582 & 0.536 & 0.207 \\
MARE $(\%)$ & 0.731 & 0.959 & 0.815 \\
STDRE $(\%)$ & 1.340 & 1.519 & 1.451 \\
\hline \multicolumn{4}{c}{ Band Gaps } \\
\hline ME $(\mathrm{eV})$ & -0.306 & -0.044 & -0.121 \\
MAE $(\mathrm{eV})$ & 0.371 & 0.329 & 0.331 \\
STDE $(\mathrm{eV})$ & 0.410 & 0.436 & 0.443 \\
MRE $(\%)$ & -4.144 & 14.917 & 11.202 \\
MARE $(\%)$ & 15.878 & 24.934 & 23.392 \\
STDRE $(\%)$ & 26.005 & 58.680 & 56.653 \\
\hline \hline
\end{tabular}

late those are as follows,

$$
\begin{aligned}
M E & =\frac{1}{N} \sum_{i=1}^{N}\left(Y_{i}-y_{i}\right) \\
M A E & =\frac{1}{N} \sum_{i=1}^{N}\left|Y_{i}-y_{i}\right| \\
M A R E & =\sum_{i}^{N}\left|Y_{i}-y_{i}\right| /\left|y_{i}\right| \\
S T D R E & =\left[\sum_{i}^{N}\left(Y_{i} / y_{i}\right)-\frac{1}{N}\left(Y_{i} / y_{i}\right)\right]^{2},
\end{aligned}
$$

where $Y_{i}$ and $y_{i}$ are the calculated and experimental values respectively. 


\section{RESULTS AND DISCUSSIONS}

\section{A. Lattice Constants}

The fundamental test one should perform to check the robustness of a given functional for solids is the equilibrium lattice constant. Predicting the accurate equilibrium lattice constant is paramount important in view of the structural properties of a solid. To perform the benchmark calculation of SRSH-TM-TPSSc and SRSHTM we employ the two functionals for studying 47 crystalline structures which includes a test set of six metals like $\mathrm{Ag}, \mathrm{Al}, \mathrm{Cu}, \mathrm{Pd}, \mathrm{K}, \mathrm{Li}$ and 41 semiconductors. Among the semiconductors we consider (i) 3 diamond structures - C, Si and Ge, (ii) 23 zinc blende structures - SiC, BN, BP, BAs, BSb, AlP, AlAs, AlSb, $\beta-\mathrm{GaN}$, GaP, GaAs, GaSb, InP, InAs, InSb, ZnS, ZnSe, ZnTe, CdS, CdSe, CdTe, MgS, MgTe, (iii) 17 ionic crystals $\mathrm{MgO}$, MgSe, CaS, CaSe, CaTe, SrS, SrSe, SrTe, BaS, BaSe, BaTe, LiCl, LiF, NaCl, LiF, NaCl, NaF. To test the performance and robustness of the SRSH-TM-TPSSc and SRSH-TM we also put HSE06 into comparison. All the hybrid functional calculations are performed starting from the well converged wavefunction of $\mathrm{PBE}$ calculation. The $\Gamma$ - centered Monkhorst-Pack ${ }^{52}$ like $11 \times 11 \times 11$ $\mathbf{k}$ grids are used for all our calculations.

In Table-I, we have summarized the performance of all the function under study. First we discuss the performance of HSE06 functional. The HSE06 functional is based on the semilocal PBE functional. Mixing fraction of exact exchange seems to be improve the lattice constant compared to its semilocal form as it is shown in reference ${ }^{53}$. It is well known that HSE06 has the inherit tendency to overestimate lattice constants and it can be overcome using a improved description of PBE i.e, PBEsol and its hybrid version HSEso ${ }^{54}$. In TABLE III we have listed the overall statistics of HSE06 for all the solids using HSE06 functional. Overall, using HSE06 we obtain the MAE of $0.039 \AA$.

Now we come to the performance of newly constructed SRSH-TM-TPSSc and SRSH-TM. Regarding the performance of SRSH-TM-TPSSc, due to the TPSS correlation the lattice constants of all the crystalline structures are overestimated in this case. This drawback can be explained from the performance of TM-TPSS as reported in reference $e^{37}$. In the reference $\frac{37}{3}$ it is shown that MAE of TM-TPSS is $0.045 \AA$. Mixing HF with TM-TPSS actually overestimates more and give the MAE of $0.045 \AA$. In the present formalism of screened hybrid functional theory using TM correlation with HF improves its over SRSH-TM-TPSSc and gives MAE $0.042 \AA$ which is only $0.004 \AA$ and $0.003 \AA$ greater than its base functional TM and hybrid HSE06 respectively. Regarding the performance of HSE06 and semilocal TM functional both perform equivalently as it is shown in this paper and reference 37 . From Fig. (11) it is evident that in case of Ge, AlAs, GaP, GaAs, InP, InAs, InSb, ZnS, ZnSe, ZnTe, CdTe, MgS, MgSe, MgTe, Ag, Cu, K the SRSH-TM ac- tually performs better than HSE06. For other cases the performance of HSE06 is better or equivalent compared to SRSH-TM.

\section{B. Band Gaps}

It is well known that the accurate band gap prediction is only achievable through the hybrid functional scheme due to the inclusion of HF exchange which actually balance the delocalization and localization problem arises from semilocal and HF exchange. As present range separated scheme is based on the hybrid interface, therefore, it is always interesting to check the performance of the present scheme for band gaps problem. Besides the present scheme is based on the semilocal funcional which is the second best performer after SCAN metaGGA in predicting the band gaps within semilocal formalism 37 . Also, it is noteworthy to mentioned that the meta-GGA functional implemented with the framework of gKS formalism gives more realistic band gap $\underline{88}$. Therefore, within meta-GGA hybrids the improvement in band gap comes from both the semilocal formalism and mixing of HF exchange.

Here, we choose 34 semiconductors (including insulators) to assess the performance of SRSH-TM-TPSSc and SRSH-TM along with the HSE06. We report performance of all the functionals in TABLE II. Here, all the band gaps are calculated at experimental lattice constants. The experimental geometries are collected from reference $\frac{73}{}$. From TABLE II it is evident that all the screened hybrid functionals perform better than their respective semilocal form due to the inclusion of HF exchange. It is well known that, the HSE06 functional is widely used functional for prediction band gap of semiconductor for small band gap material (upto $5 \mathrm{eV}$ ). For large band gap materials HSE06 actually underestimates the band gap. But, the performance of HSE06 quite productive because the computational cose of HSE06 is less than accurate many body treatment like GW or many body perturbation theory (MBPT). Regarding the performance of meta-GGA level screened range separated hybrids SRSH-TM-TPSSc and SRSH-TM, we observed the band gap is more enhanced than HSE06 for all the materials. this is obvious because the meta-GGA functionals are implemented in gKS scheme which produced more realistic band gap than GGA. Here, mixing the HF exchange with meta-GGA level semilocal functionals actually enhance the band gap more. For the semiconductors for which HSE06 underestimates the band gap slightly, inclusion of HF exchange with meta-GGA level screened range separation scheme actually compensate those. Regarding the overall comparison of SRSHTM-TPSSc with SRSH-TM and HSE06, the SRSH-TMTPSSc overestimate the band gaps of those materials for which HSE06 and SRSH-TM quite accurate. It has been observed for the band gap values of $\mathrm{ScN}, \mathrm{Si}, \mathrm{Ge}, \mathrm{SiC}$, GaP, InP, InAs, InSb, and CdTe that HSE06 is accurate 
for those systems. SRSH-TM also gives very comparable results with HSE06. But, the overestimation of band gaps is observed using SRSH-TM-TPSSc for those materials. Overall, the SRSH-TM functional is quite productive over HSE06 for predicting the band gap of semiconductor materials. It is also noteworthy to mention that, the calculated band gap values at their experimental lattice constant can also be compared with different semilocal functionals which are quite good (but not always) in predicting band gaps in semilocal level ${ }^{73}$. Interestingly, few band gaps reported using TPSS based screened hybrid functionals in reference $\frac{46}{}$ is also comparable with the band gap of SRSH-TM in TABLE II.

The only drawback of present functional form is that it overestimates the band gap for which HSE06 is exact (or slightly overestimating). It could be avoided by using the full reverse engineered exchange hole of TM functional. It also noteworthy that TPSS based screened functional also overestimates those values as shown for few specific cases in reference $\underline{46}$.

\section{CONCLUSIONS AND FUTURE DIRECTION}

We assess the performance of solid state lattice constants and band gaps using the meta-GGA level screened range separated hybrids SRSH-TM-TPSSc and SRSHTM with the PAW method. To check the robustness of the present proposition we also compared the performance of the present functional with HSE06 functional. The results obtained using the SRSH-TM-TPSSc and SRSH-TM are found to be interesting. This is first ever test of any meta-GGA level screened range separated hybrid functional for both the lattice constants and band gaps. From the prospective of lattice constants the overall performance of SRSH-TM is quite impressive. The MAE of SRSH-TM differs from HSE06 and TM semilocal functional only by $0.003 \AA$ and $0.004 \AA$. It has been observed that for several cases where HSE06 has the tendency to overestimate the lattice constants SRSHTM performs quite well. Regarding the performance of SRSH-TM and SRSH-TM-TPSSc, the performance of SRSH-TM is quite better compare to SRSH-TM-TPSSc. The improved performance of SRSH-TM actually comes from change is correlation. TM exchange coupled with TM correlation performs better than TPSS correlation for solid state lattice constants.

From the point of view of functional form it is very simple. Only the LDA based exchange hole is used in its short range together with $\mathrm{HF}$ short range exchange. It is observed that a simple modification on the top of the TM functional improves its performance for band gap. As discussed earlier the band gap problem is not achievable through the semilocal level only suitably mixing HF with semilocal performs well for band gaps. The performer of SRSH-TM indicates that it is a good competitor with HSE06, especially for cases HSE06 has the tendency to underestimate the band gaps. Though few cases SRSHTM overestimates the band gap more compare to HSE06. But the overall performance of SRSH-TM is quite well.

Lastly, we want to conclude that the present SRSH functional based on TM semilocal functional keeps all the good properties of TM functionals which is very accurate for predicting solid state properties in semilocal level. The advantage of the present SRSH-TM (we recommand SRSH-TM over SRSH-TM-TPSSc because SRSH-TM performances more balanced way than SRSHTM-TPSSc for both the lattice constant and band gap) is that is mixes HF which actually overcome several drawback that is actually not achievable in semilocal level for TM, more precisely the band gap. Another interesting feature of the present formalism is that it is based on meta-GGA level theory which is very accurate than GGA in different ways. Several electronic structural properties can be studied using this screened meta-GGA level functional. As a future direction of present SRSH functional it is always interesting to study the dielectric dependent performance of SRSH because it improves the screening effects and several other properties ${ }^{74}-77$.
* subrata.jana@niser.ac.in

1 W. Kohn and L. J. Sham, Phys. Rev. 140, A1133 (1965).

2 Density Functional Theory: An Advanced Course, E. Engel and R. M. Dreizler, Springer (2011).

3 J. P. Perdew and K. Schmidt, AIP Conference Proceedings 577, 1 (2001).

4 J. P. Perdew and A. Zunger. Phys. Rev. B, 23 5048, (1981).

5 J. P. Perdew and Y. Wang, Phys. Rev. B 33, 8800 (1986).

6 A. D. Becke, Phys. Rev. A 38, 3098 (1988).

7 C. Lee, W. Yang, and R. G. Parr, Phys. Rev. B 37, 785 (1988).

8 J. P. Perdew, J. A. Chevary, S. H. Vosko, K. A. Jackson, M. R. Pederson, D. J. Singh, and C. Fiolhais, Phys. Rev. B 46, 6671 (1992).

9 J. P. Perdew, K. Burke, and M. Ernzerhof, Phys. Rev. Lett. 77, 3865 (1996).
10 R. Armiento and A. E. Mattsson, Phys. Rev. B 72, 085108 (2005).

11 Z. Wu and R. E. Cohen, Phys. Rev. B 73, 235116 (2006).

12 J. P. Perdew, A. Ruzsinszky, G. I. Csonka, O. A. Vydrov, G. E. Scuseria, L. A. Constantin, X. Zhou, and K. Burke, Phys. Rev. Lett. 100, 136406 (2008).

13 L. A. Constantin, J. P. Perdew, and J. M. Pitarke, Phys. Rev. B 79, 075126 (2009).

14 E. Fabiano, L. A. Constantin, and F. Della Sala, Phys. Rev. B 82, 113104 (2010).

15 E. Fabiano, L. A. Constantin, and F. Della Sala, J. Chem. Theory Comput., 7 (11), pp 35483559 (2011).

16 L. A. Constantin, A. Terentjevs, F. Della Sala, P. Cortona, and E. Fabiano, Phys. Rev. B 93, 045126 (2016).

17 A. D. Becke and M. R. Roussel, Phys. Rev. A 39, 3761 (1989). 
18 T. V. Voorhis and G. E. Scuseria, J. Chem. Phys. 109, 400 (1998).

19 F. A. Hamprecht, A. J. Cohen, D. J. Tozer, and N. C. Handy, J. Chem. Phys. 109, 6264 (1998).

20 M. Ernzerhof and G. E. Scuseria, J. Chem. Phys. 110, 5029 (1999).

${ }^{21}$ Y. Zhao and D. G. Truhlar, J. Chem. Phys. 125, 194101 (2006)

22 J. Tao, J. P. Perdew, V. N. Staroverov, and G. E. Scuseria, Phys. Rev. Lett. 91, 146401 (2003).

23 J. P. Perdew, A. Ruzsinszky, G. I. Csonka, L. A. Constantin, and J. Sun, Phys. Rev. Lett. 103, 026403 (2009).

24 L. A. Constantin, E. Fabiano, and F. Della Sala, J. Chem. Theory Comput., 9 (5), pp 22562263 (2013).

25 J. Sun, A. Ruzsinszky, and J. P. Perdew, Phys. Rev. Lett. 115, $036402(2015)$

26 J. Tao and Y. Mo, Phys. Rev. Lett. 117, 073001 (2016).

27 P. Hao, J. Sun, B. Xiao, A. Ruzsinszky, G. I. Csonka, J. Tao, S. Glindmeyer, and J. P. Perdew, J. Chem. Theory Comput. 9, 355 (2013).

28 Y. Mo, G. Tian and J. Tao, Phys. Chem. Chem. Phys., .19, 21707-21713 (2017)

29 J. Paier, R. Hirschl, M. Marsman, G. Kresse, J. Chem. Phys. 122, 234102 (2005).

30 P. Haas, F. Tran, and P. Blaha, Phys. Rev. B 79, 085104 (2009).

31 F. Tran, J. Stelzl, and P. Blaha, J. Chem. Phys. 144, 204120 (2016).

32 Y. Mo, G. Tian, R. Car, V. N. Staroverov, G. E. Scuseria, and J. Tao, Phys. Rev. B 95, 035118 (2017).

${ }^{33}$ Y. Mo, G. Tian and J. Tao, Chem. Phys. Lett. 628, 38-42 (2017).

34 A. E. Mattsson, R. Armiento, J. Paier, G. Kresse, J. M. Wills, and T. R. Mattsson, J. Chem. Phys. 128, 084714 (2008).

35 J. Sun, M. Marsman, A. Ruzsinszky, G. Kresse, and J. P. Perdew Phys. Rev. B 83, 121410(R) (2011).

36 J. Sun, M. Marsman, G. I. Csonka, A. Ruzsinszky, P. Hao, Y. S. Kim, G. Kresse, and J. P. Perdew, Phys. Rev. B 84, 035117 (2011).

37 S. Jana, A. Patra and P. Samal (submitted).

38 A. J. Cohen, P. Mori-Sanchez, and W. Yang, Chem.Rev. 112, 289 (2012).

39 A. D. Becke, J. Chem. Phys. 104, 1040 (1996).

40 P. J. Stephens, F. J. Devlin, C. F. Chabalowski, and M. J. Frisch, J. Phys. Chem. 98, 11623 (1994).

41 V. N. Staroverov, G. E. Scuseria, J. Tao, and J. P. Perdew, J. Chem. Phys. 119, 12129 (2003); 121, 11507(E) (2004).

42 J. Heyd, G. E. Scuseria, and M. Ernzerhof, J. Chem. Phys. 118, 8207 (2003).

43 A. V. Krukau, O. A. Vydrov, A. F. Izmaylov, and G. E. Scuseria, J. Chem. Phys. 125, 224106 (2006).

44 T. Yanai, D. P. Tew, and N. C. Handy, Chem. Phys. Lett. 393 (2004) 5157.

45 O. A. Vydrov, and G. E. Scuseria, J. Chem. Phys. 125, 234109 (2006).

46 J. Tao, I. W. Bulik, and G.E. Scuseria, Phys. Rev. B 95, 125115 (2017).

47 A. Savin, in Recent Developments and Applications of Modern Density Functional Theory, Ed. J. M. Seminario (Elsevier, Amsterdam, 1996), p. 327.

48 H. Iikura, T. Tsuneda, T. Yanai and K. Hirao, J. Chem. Phys. 115, 3540 (2001).

49 B. Patra, S. Jana and P. Samal, Phys. Chem. Chem. Phys.,
(2018) (accepted) DOI: 10.1039/C8CP00717A.

50 S. Jana and P. Samal, Phys. Chem. Chem. Phys., (2018) (accepted) DOI: 10.1039/C8CP00333E.

51 J. Paier, M. Marsman, and G. Kresse, Journal of Chemical Physics 127, 024103 (2007).

52 M. Marsman, J. Paier, A. Stroppa, G. Kresse Journal of Physics: Condensed Matter 20 (6), 064201 (2008).

53 J. Paiera, M. Marsman, K. Hummer, G. Kresse I. C. Gerber and J. G. ngyn, J. Chem. Phys. 124, 154709 (2006).

${ }^{54}$ L. Schimkaa, J. Harl, and G. Kresse J. Chem. Phys. 134, 024116 (2011).

55 J. Heyd, J. E. Peralta, G. E. Scuseria and R. L. Martin, J. Chem. Phys. 123, 174101 (2005).

${ }^{56}$ F. Oba, A. Togo, I. Tanaka, J. Paier and G. Kresse Phys. Rev. B 77 (24), 245202 (2008).

57 J. L. F. Da Silva, M. V. Ganduglia-Pirovano, J. Sauer, V. Bayer and G. Kresse, Physical Review B 75 (4), 045121 (2007)

58 A. Janotti, J. B. Varley, P. Rinke, N. Umezawa, G. Kresse, C. G. Van de Walle Phys. Rev. B 81 (8), 085212 (2010).

59 J. Paier, M. Marsman, G. Kresse, Phys. Rev. B 78 (12), 121201 (2008)

60 Y. S. Kim, M. Marsman, G. Kresse, F. Tran and P. Blaha Phys. Rev. B 82 (20), 205212 (2010)

61 A. Stroppa, G. Kresse New Journal of Physics 10 (6), 063020 (2008).

62 C. Franchini, R. Podloucky, J. Paier, M. Marsman, G. Kresse, Physical Review B 75 (19), 195128 (2007).

63 R. Wahl, D. Vogtenhuber, G. Kresse Phys. Rev. B 78 (10), 104116 (2008).

64 A. Stroppa, K. Termentzidis, J. Paier, G. Kresse and J. Hafner Phys. Rev. B 76 (19), 195440 (2007)

65 Y. S. Kim, K. Hummer, G. Kresse Phys. Rev. B 80 (3), 035203 (2009).

66 K. Hummer, J. Harl, G. Kresse Phys. Rev. B 80 (11), 115205103 (2009).

67 I. C. Gerber, J. G. ngyn, M. Marsmana and G. Kresse The Journal of chemical physics 127 (5), 054101 (2007).

68 J. Heyd, G. E. Scuseria The Journal of chemical physics 121 (3), 1187-1192 (2004).

69 J. Heyd, J. E. Peralta, G. E. Scuseria, R. L. Martin The Journal of chemical physics 123 (17), 174101 (2005)

70 T. M. Henderson, B. G. Janesko, G. E. Scuseria The Journal of chemical physics 128 (19), 194105 (2008).

71 J. E Peralta, J. Heyd, G. E. Scuseria, R. L. Martin Physical Review B 74 (7), 073101 (2006).

72 E. N. Brothers, A. F. Izmaylov, J. O. Normand, V. Barone, G. E. Scuseria, The Journal of chemical physics 129 (1), 011102 (2008).

73 F. Tran and P. Blaha, J. Phys. Chem. A, 121 (17), pp 33183325 (2017).

74 J. H. Skone, M. Govoni, G. Galli Physical Review B 89 (19), 195112 (2014)

75 N. P. Brawand, M. Vörös, M. Govoni, G. Galli, Physical Review X 6 (4), 041002 (2016)

76 J. H. Skone, M. Govoni, G. Galli Physical Review B 93 (23), 235106 (2016).

77 N. P. Brawand, M. Govoni, M. Vörös, G. Galli, Journal of chemical theory and computation 13 (7), 3318-3325 (2017).

78 L. A. Constantin, J. P. Perdew, and J. Tao, Phys. Rev. B, 2006, 73, 205104.

79 Lucian A. Constantin, Eduardo Fabiano, and Fabio Della Sala, Phys. Rev. B 88, 125112 (2013).

${ }^{80}$ Lucian A. Constantin,1 John P. Perdew, and J. M. Pitarke, 
Phys. Rev. B 79, 075126 (2009)

81 P. E. Blöchl, Phys. Rev. B, 50:17953, (1994).

82 G. Kresse and D. Joubert, Phys. Rev. 59 , 1758 (1999).

83 G. Kresse and J. Hafner, Phys. Rev. B 47 , 558 (1993); ibid. 49,14251 (1994).

${ }^{84}$ G. Kresse and J. FurthmAller, Comput. Mat. Sci. 6 , 15 (1996).

${ }^{85}$ G. Kresse and J. FurthmAller, Phys. Rev. B 54, 11169
(1996)

${ }^{86}$ G. Kresse and J. Hafner, J. Phys.: Condens. Matt. 6, 8245 (1994).

87 H. J. Monkhorst and J. D. Pack, Phys. Rev. B 13, 5188 (1976).

88 Zeng-hui Yang, H. Peng, J. Sun, and J. P. Perdew, Phys. Rev. B 93, 205205 (2016). 